\title{
Cheap, Versatile, and Turnkey Fabrication of Microfluidic Master Molds Using Consumer Grade LCD Stereolithography 3D Printing
}

\section{Vincent G. Colin}

Angers University: Universite d'Angers

Théo A. Travers

Angers University: Universite d'Angers

Denis Gindre

Angers University: Universite d'Angers

Régis Barillé

Angers University: Universite d'Angers

Matthieu Loumaigne ( $\nabla$ matthieu.loumaigne@univ-angers.fr )

University of Angers https://orcid.org/0000-0002-7396-9352

\section{Research Article}

Keywords: microfabrication, micro uidics, dry Im photoresist, low-cost, gradient mixer

Posted Date: March 17th, 2021

DOI: https://doi.org/10.21203/rs.3.rs-293330/v1

License: (9) This work is licensed under a Creative Commons Attribution 4.0 International License.

Read Full License 


\title{
Cheap, versatile, and turnkey fabrication of microfluidic master molds using consumer grade LCD stereolithography 3D printing
}

\author{
Vincent G. Colin ${ }^{1}$. Théo Travers ${ }^{1}$. Denis Gindre ${ }^{1}$. Régis Barillé ${ }^{1}$. \\ Matthieu Loumaigne ${ }^{1, *}$
}

Received: date / Accepted: date

\begin{abstract}
The recent development of $3 \mathrm{D}$ printers allowed a lot of limitations in the field of microfabrication to be circumvented. The ever-growing chase for smaller dimensions has come to an end in domains such as microfluidics, and the focus now shifted to a cost-efficiency challenge. In this paper, the use of a high-resolution stereolithography LCD 3D printer is investigated for fast and cheap production of microfluidic master molds. More precisely, the UV LED array and the LCD matrix of the printer act as an illuminator and a programmable photomask for soft lithography. The achieved resolution of around $100 \mu \mathrm{m}$ is mainly limited by the pixel geometry of the LCD matrix. A tree-shape gradient mixer was fabricated using the presented method. It shows very good performances despite the presence of sidewall ripples due to the uneven pixel geometry of the LCD matrix. Given its sub-€1,000 cost, this method is a very good entry point for labs wishing to explore the potential of microfluidic devices in their experiments, as well as a teaching tool for introducing students to microfluidics.
\end{abstract}

Keywords microfabrication · microfluidics · dry film photoresist $\cdot$ low-cost $\cdot$ gradient mixer

\section{Introduction}

Despite its quite recent development, microfluidics is nowadays seen as much as a tool as its own research field [1. There is no doubt about the relevance of such an implement for specific studies (lab on chip 2], bio 3] 1

Laboratoire MOLTECH-Anjou, UMR CNRS 6200, UNIV Angers, SFR MATRIX, 2 Bd Lavoisier, 49045 Angers CEDEX, France

E-mail: matthieu.loumaigne@univ-angers.fr and chemical analysis 4, etc.). Sub-micrometric precision was reached using clean rooms and specific materials such as SU-8 resin. However, this process can be very expensive, and not accessible in every lab 5 . Moreover, not all applications require the finest resolutions possible, and it is more relevant in various cases to engineer cheaper microfluidic chips 6 . The focus is rather being drawn on the variety of circuits that can be engineered and processed in a short time period [7]. Different methods have been investigated and developed over the recent years, with a focus on lowering the production costs of such devices, in order to better integrate them into regular diagnosis 8 .

In this article, the process of design and fabrication of microfluidic master molds using a consumer grade stereolithography (SLA) LCD 3D printer is presented. The LED array of the 3D printer delivers a parallel beam used to insolate a dry film photoresist through the LCD screen of the same printer. The whole process can be described as turnkey ; the consumer-grade device can be used without any required modification. The design is directly displayed on the LCD screen, and no additional optics are required in order to shape the insolation beam.

The process described here is suited for prototyping, since the printed patterns can be modified at will quickly. The entire process from start to finish requires less than 6 hours to manufacture a microfluidic device. Although the resulting devices are not on par in term of resolution with the state of the art, the idea here is a cheap and quick alternative, either to investigate different patterns for microfluidic devices, or even as an academic tool in order to introduce students to the subject of microfluidics. The process of microfabrication will be extensively described in the first part, and results obtained for a specific application of a tree-shaped 
gradient mixer will be presented in the second part to demonstrate the feasibility of the method. Comparisons with other methods sharing the same philosophy will be carried out in a third part.

\section{Material and methods}

The manufacturing of the microfluidic circuits is conducted in a dark, non clean room setting. The goal is to demonstrate the feasibility of such microchips outside of the state of the art, and in very controlled conditions. The experimental environment described here can be reproduced in basically any room.

\subsection{Microfluidic chips design}

The microfluidic devices were initially designed with a 3D CAD software (OpenSCAD), and sliced through the software provided by the printer. However, it is also possible to draw the printed pattern at the pixel level by uploading directly a bitmap file instead of a CAD file (see SI section 1). The devices were then designed with a generic raster graphic editor.

\subsection{Substrate preparation}

50 um-thickness Ordyl FP450 dryfilm negative photoresist is used as the base material to create the microfluidic master mold. The theoretical achievable transverse resolution using this material is $25 \mu \mathrm{m}$. PET (Polyethylene terephthalate) plates were chosen as a substrate for the dryfilm photoresist, because they are easier to process rather than glass slides, and offer better adherence to the dryfilm photoresist 9. The only plate size limitation is the printing dimension, which is constrained by the size of the LCD screen, roughly $12 \times 7 \mathrm{~cm}^{2}$. The pressing of the film against the PET substrate is an important step carried out with a basic thermal laminator (FGK-220). This step determines the quality of the microchip master mold. A particular attention has to be paid to avoid the formation of wrinkles between the dry film and the PET substrate, which could alterate the characteristics of the final microchannel. Additional practical details are given in section SI 2.

\subsection{Insolation through a SLA LCD 3D printer}

The printer used for insolation is a $€ 1,500$ Phrozen Shuffle $4 \mathrm{~K}$ 10. It is sold as a high resolution SLA 3D printer, initially designed to work with liquid photosensitive resins. The idea is to create a $3 \mathrm{D}$ object by printing successive layers on top of each other. Originally, a laser was used as the light source; nowadays, high resolution LCD screens are extensively used, in combination with a high power LED array, in order to dramatically lower the prices of such apparatus.

The theoretical achievable transverse resolution is $31 \mu \mathrm{m}$. The printer uses a 3x5 matrix of $405 \mathrm{~nm}$ LEDs which is roughly collimated with one lens per LED. The light is then selectively masked by a $2160 \times 3840$ " $4 \mathrm{~K}$ " LCD screen. The whole system is operated by a Raspberry Pi and a custom motherboard.

The typical insolation time to ensure the polymerization of the dryfilm takes between 10 and 15 minutes, depending on the size of the details to be printed. Furthermore, thanks to the large size of the LCD screen, up to 5 microscope slides can be inserted at the same time in the insolation chamber, making the parallelization task incredibly easier.

The development process can be initiated as soon as 15 minutes after the illumination step is over. The substrates are submerged in a $\mathrm{K}_{2} \mathrm{CO}_{3}$ solution (1\% weight concentration), at a temperature of around $20^{\circ} \mathrm{C}$. The development time takes usually 2 minutes, but highly depends on the size of the finest details of the circuit. The obtained microfluidic master molds are stored in dry and dark conditions, and protected from the dust in plastic Petri dishes.

\subsection{PDMS imprint}

A negative replica of the master mold can then be created with PDMS. Sylgaard 184 prepolymer is strongly mixed with the corresponding curing agent with a weight ratio of $10: 1$. This mixture is poured on the master mold, subjected to vacuum $\left(10^{-3}\right.$ bar $)$ for a few minutes in order to eliminate air bubbles, and then left to dry in an oven for a minimum of 2 hours at $60^{\circ} \mathrm{C}$. Higher temperatures would imply faster drying of the PDMS, however, it tends to destroy and melt the photoresist, resulting in a deterioration of the PDMS imprint.

To achieve a sealed microfluidic circuit, the PDMS imprint is bonded to a glass microscope slide using a corona treater 11 .

\subsection{Characterization apparatus}

Height measurements of the master mold channels were obtained with a Veeco Dektak 6M Profilometer. 
2.6 Microfluidics setup and absorbance measurement

The microfluidics setup is built around an IX73 Olympus inverted microscope.

The pressure in the channels is controlled using an Elveflow microfluidic flow controller OB1 MK3+ and the flow rates are measured via MFS2 and MFS3 Elveflow flow sensors.

The Rhodamine 6G dye (Sigma-Aldricht CAS: 98938-8) used for absorbance measurement was prepared at $45 \mathrm{mg} \mathrm{L}^{-1}$ in ultrapure water.

The absorbance measurements are performed directly in the microfluidics channel according to the protocol given by Werts et al. [12. The light from a day light white high-power LED lamp (Thorlabs SOLIS-3C), is collimated and focused onto the microfluidic device, and then collected through a x4 0.1 NA microscope objective. The optical beam goes through a 550LP optical filter in order to eliminate the fluorescence of the Rhodamine $6 \mathrm{G}$. The image of the channel is then formed on a color camera (Canon EOS 70D). The absorbance was measured using the green channel of the camera. The baseline signal $I 0$ was obtained by delimitation of a region of interest (RI) in the PDMS areas outside the microfluidic channels. The signal $I$ was obtained by the mean value of the intensity inside the channel and the absorbance $A$ was computed as $A=-\log _{10}\left(I / I_{0}\right)$. According to the Beer-Lambert law, at low absorbance, the absorbance is proportional to the concentration of Rhodamine 6G.

\section{Results}

\subsection{Characterisation of the LED array}

The central wavelength of the emitting LED is at $405 \mathrm{~nm}$ with an approximate width of $15 \mathrm{~nm}$ (see SI S3). The luminance provided by the LED matrix is not uniform (see picture on figure SI 4). The optical power was estimated between $1.6 \mathrm{~mW} \mathrm{~cm}^{-2}$ and $2.2 \mathrm{~mW} \mathrm{~cm}^{-2}$ (see SI section 5). As a comparison, the data sheet of the dry film photoresist recommends an optimal energy of exposure of $300 \mathrm{~mJ} \mathrm{~cm}^{-2}$ to $350 \mathrm{~mJ} \mathrm{~cm}^{-2}$ for a UV source between $360 \mathrm{~nm}$ and $380 \mathrm{~nm}$. Consequently, assuming that the absorption of the photoresist is lower at $405 \mathrm{~nm}$ than $370 \mathrm{~nm}$, an optical power of $2 \mathrm{~mW} \mathrm{~cm}^{-2}$ is compatible with the $600 \mathrm{~s}$ exposure at $405 \mathrm{~nm}$ used here to insolate the photoresist.

The light emitted by the 405 LED array is not well collimated. In fact, it seems that the LED array aims at having a high luminance and quite uniform lighting at the cost of a parallel beam. Indeed, the matrix

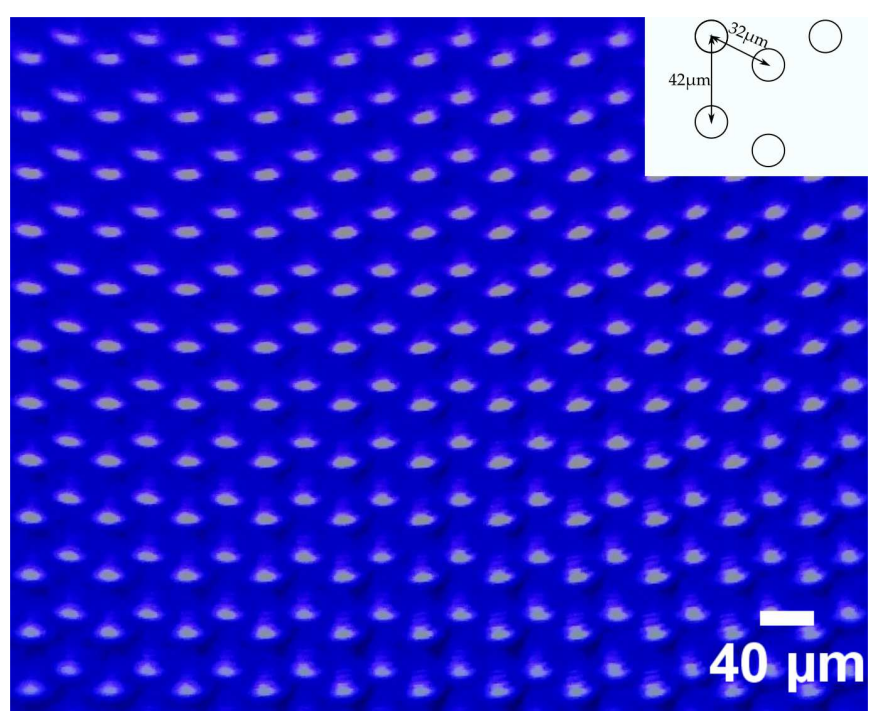

Fig. 1 Image of the LCD matrix used for illuminating the photoresist film. Inset : schematic of the pixel geometry of the LCD matrix.

uses quite a large LED chip with a relatively small spatial coherence which prevents it from being efficiently collimated with a lens. Quantifying the beam divergence is difficult since it appears that different positions on the LED array have different angles of divergence/convergence.

\subsection{Characterisation of the LCD matrix}

A close picture of the LCD matrix, obtained with a handheld microscope, is shown on the figure 11. The picture shows clearly that the pixels are organized in a non uniform shifted pattern. More specifically, pixels form vertical lines which are each shifted by $33 \%$ of the vertical inter-pixel distance compared to the next line. As discussed below, this uneven pixel geometry is the main obstacle to achieving the best isotropic and finest resolution. We present in section 3.4 a very simple method to mitigate this effect, even though this method does not balance the precision of vertical and horizontal patterns.

\subsection{Single microfluidic channel master mold and maximum resolution}

Figures 2 a), b), c) and d) show optical images of the master mold of vertical channels obtained using respectively one, two, five and ten pixels of the LCD matrix. From the measurement obtained with the profilometer (fig 2 e)) we can see that the minimum channel width obtained here is $75 \mu \mathrm{m}$. This value is higher than what could have been expected with a $31 \mu \mathrm{m}$ LCD pixel size 


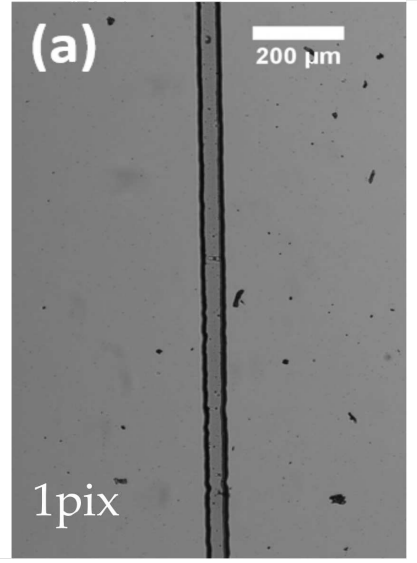

(e)

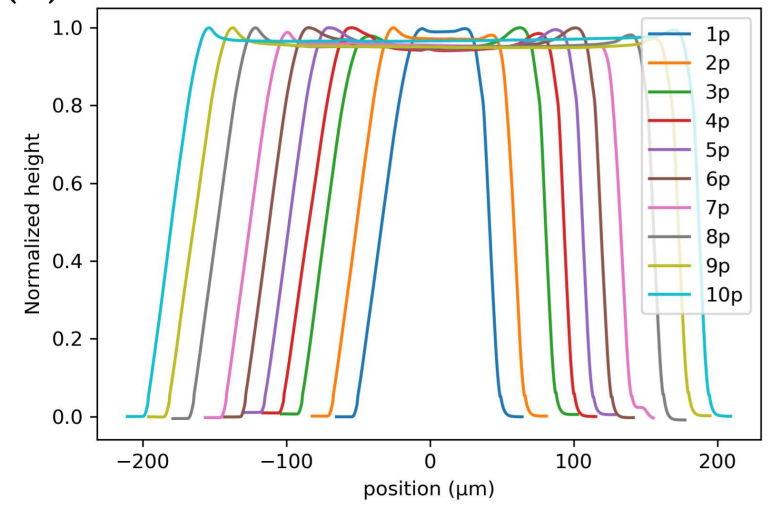

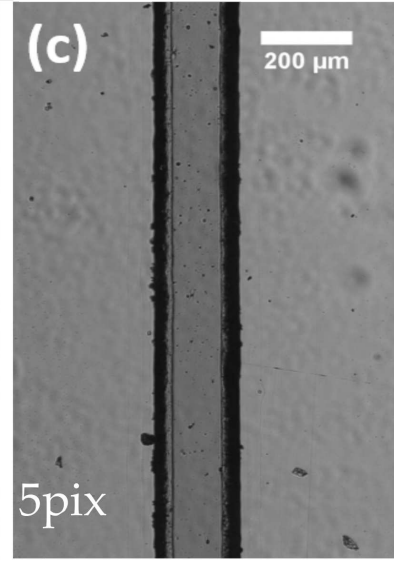

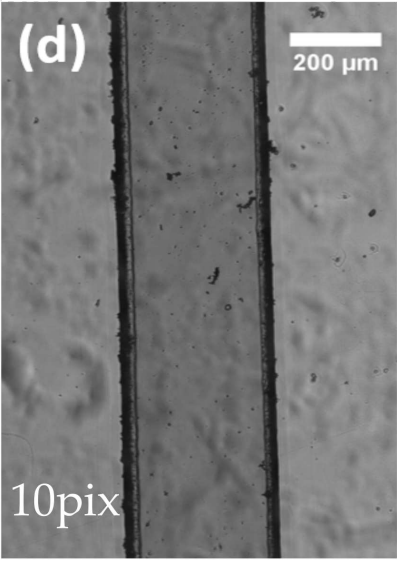

(f)

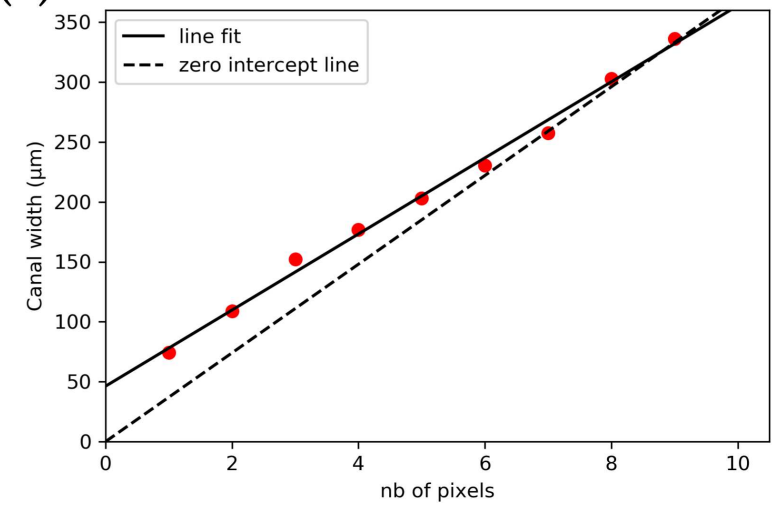

Fig. 2 a,b,c,d) Optical image of a micro-channel (vertically insolated) obtained with 1, 2, 5 and 10 pixels of the LCD screen respectively. e) Horizontal profile of micro-channels obtained for a width corresponding from 1 to 10 pixels of the LCD screen. f) Evolution of the width at half maximum of vertical micro-channels with the number of pixels on the LCD screen. The plain line takes into account all the points whereas the dashed line has a forced zero intercept and only takes into account the channels made with more than 5 pixels.

and a $25 \mu \mathrm{m}$ resolution for the dryfilm photoresist. This is most likely due to the uneven pixel geometry of the LCD screen and the non parallelism of the LED array beams.

The $75 \mu \mathrm{m}$ value potentially corrsponds to the maximum expected resolution of this setup. However, one can see some oscillations around the channel. This effect directly results from the uneven pixel geometry of the LCD screen. For a one pixel large vertical channel (see fig $2 \mathrm{a}$ and fig $3 \mathrm{a}$ ), this oscillation represents a $15 \%$ modulation of the width of the micro channel. It can also be seen on figure 2 ) that the canal width, for a channel bigger than 5 pixel, is almost proportional to the pixel size of the pattern of the LCD. One additional pixel leads to an additional width of approximately $36 \mu \mathrm{m}$ which is sightly more than the pixel size of the LCD screen.

However, drawing a single pixel vertical line is a special case. Indeed, looking at a horizontal channel (cf fig]3k), one can see stronger oscillations around the channel compared to the vertical channel. While the mean width of the canal is kept approximately constant, the position of the canal oscillates with an amplitude of roughly $30 \%$ of the canal width. Once again, this is due to the LCD screen pixel geometry. For a 5 pixel pattern on the LCD, corresponding to a $200 \mu \mathrm{m}$ channel, the oscillation represents only $7 \%$ of the micro channel.

The mean height of the micro channel is $47 \mu \mathrm{m}$, which is in agreement with the thickness of the dry film photoresist.

Additionally, the master mold obtained from square patterns is shown in SI S6 in order to qualitatively evaluate the spatial low-pass filter effect of the whole system.

\subsection{Mitigating the effect of the pixel geometry}

Firstly, it is important to mention that a thicker $125 \mu \mathrm{m}$ dryfilm photoresist (Ordyl P50125) was unsuccessfully tested. The lower resolution (125 $\mu \mathrm{m}$ transverse resolution) could have acted as a spatial low-pass filter, 


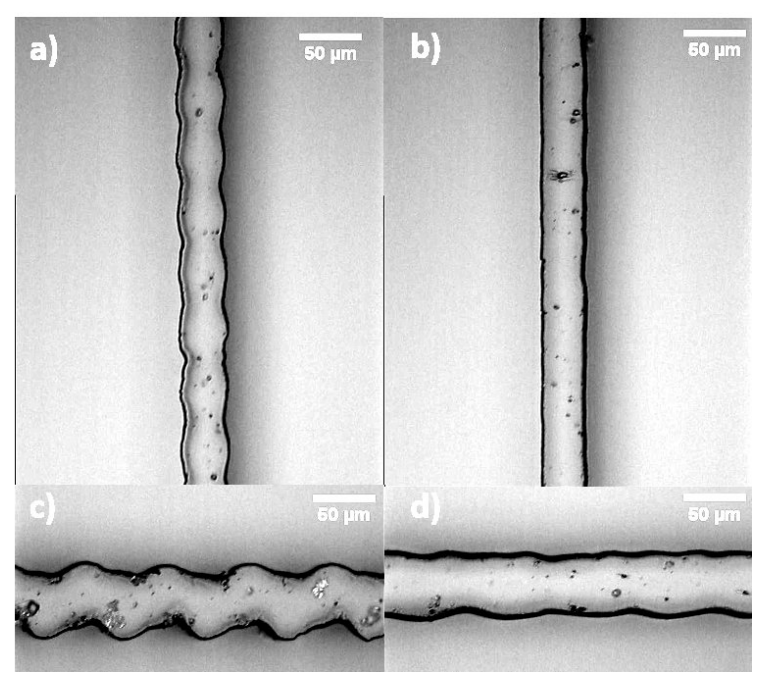

Fig. 3 Optical image of a micro-channel created from 1 pixel of a LCD screen a) vertical, pressed against the LCD screen. The small sidewall ripples may be attributed to the fact that the vertical pixels of the LCD matrix are not totally contiguous. b) vertical, insolated $340 \mu \mathrm{m}$ away from the LCD screen, c) horizontal, pressed against the LCD screen. The important sidewall ripples are most surely due to the fact that, for drawing a horizontal line, alternating pixels of two different rows are turned on by the LCD matrix. (cf fig 1). d) horizontal, insolated $340 \mu \mathrm{m}$ away from the LCD screen

smoothing the pixel geometry effect. Instead, we apparently have observed the Fresnel diffraction created by the LCD matrix inscribed in different heights of the photoresist (See SI S7).

Indeed, the LCD mask also acts as a diffraction grid. Consequently, by being sufficiently far away from the surface of the LCD matrix, the light diffracting from several pixels can be blended into a smoother shape. This effect is shown on the figure 3 where a 1-pixelmicro-channel was insolated on the resin while pressed against the LCD screen (fig. 3 a and c) or $340 \mu \mathrm{m}$ away from it (fig. 3 b and d).

\subsection{Gradient mixer}

Here are presented the results obtained with a gradient mixer. The basic tree-shape design 13 ] was deliberately chosen in order to maximise the footprint and show that large microchips can be easily manufactured. Additionally, creating a functional gradient mixer is more demanding than it seems. Indeed, the tolerance on the channel dimension is quite low since a slight imbalance in the hydrodynamic resistances of the different arms of the mixer will add up at each stage of the mixer and lead to concentrations significantly different from the theoretical values[14] (see SI S8).

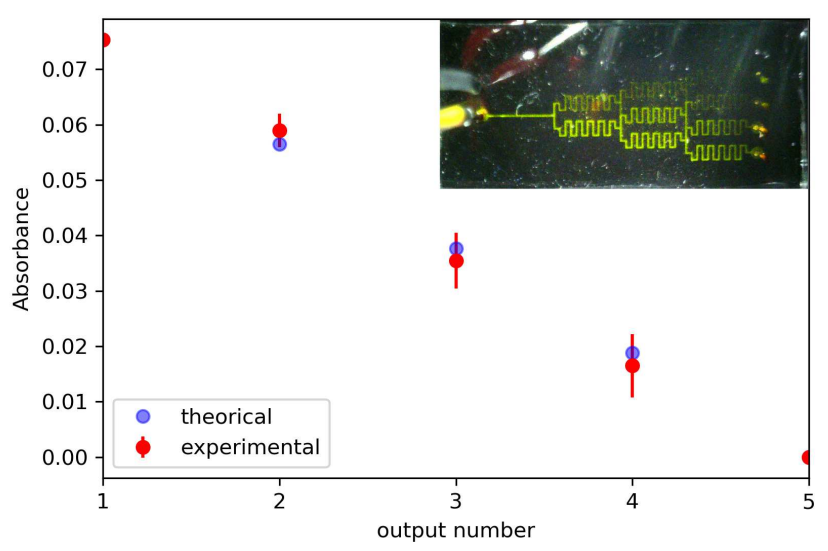

Fig. 4 Absorbance obtained at the five outputs of the gradient mixer chip after mixing Rhodamine $6 \mathrm{G}$ and water. Inset : picture of the microdevice with Rhodamine $6 \mathrm{G}$ visualised via its fluorescence.

Figure 5 shows optical images of the PDMS micro channels. Despite the apparent roughness of the microfluidic devices, in particular the sidewall ripples mentioned previously, the total hydrodynamic resistance on each channel is well balanced since applying the same pressure on the entrance with the same liquid leads to an even flow in the two channels within a $5 \%$ margin error.

Based on the dimensions and length of the microchannel, the flow rate was set to $1 \mu \mathrm{L} \mathrm{min}{ }^{-1}$ so that the rhodamine $6 \mathrm{G}$ would fully mix into water by diffusion 15 .

Inset of figure 4 is a picture of the micro-device filled with water and rhodamine $6 \mathrm{G}$ and the figure 4 shows the evolution of the absorbance of the liquid along the five outputs of the gradient mixer. Experimental output concentrations are in agreement with the theoretical ones [16].

Concerning the manufacturing aspect, the resin was insolated $340 \mu \mathrm{m}$ away from the LCD screen (i.e. the thickness of two microscopy glass coverslips). Five master molds were insolated at the same time using the 5 inch display of the 3D printer. Consequently, five PDMS chips were obtained in a matter of a few hours.

\section{Discussion}

The goal of the present paper is to demonstrate another use of a consumer-grade product in the framework of research and/or academic activities. In this part, this method will be compared in this part against other techniques, acting as cheaper and faster counterparts to the more traditional fabrication strategy using white rooms and expensive reagents. Different approaches have been studied in order to reduce the cost 

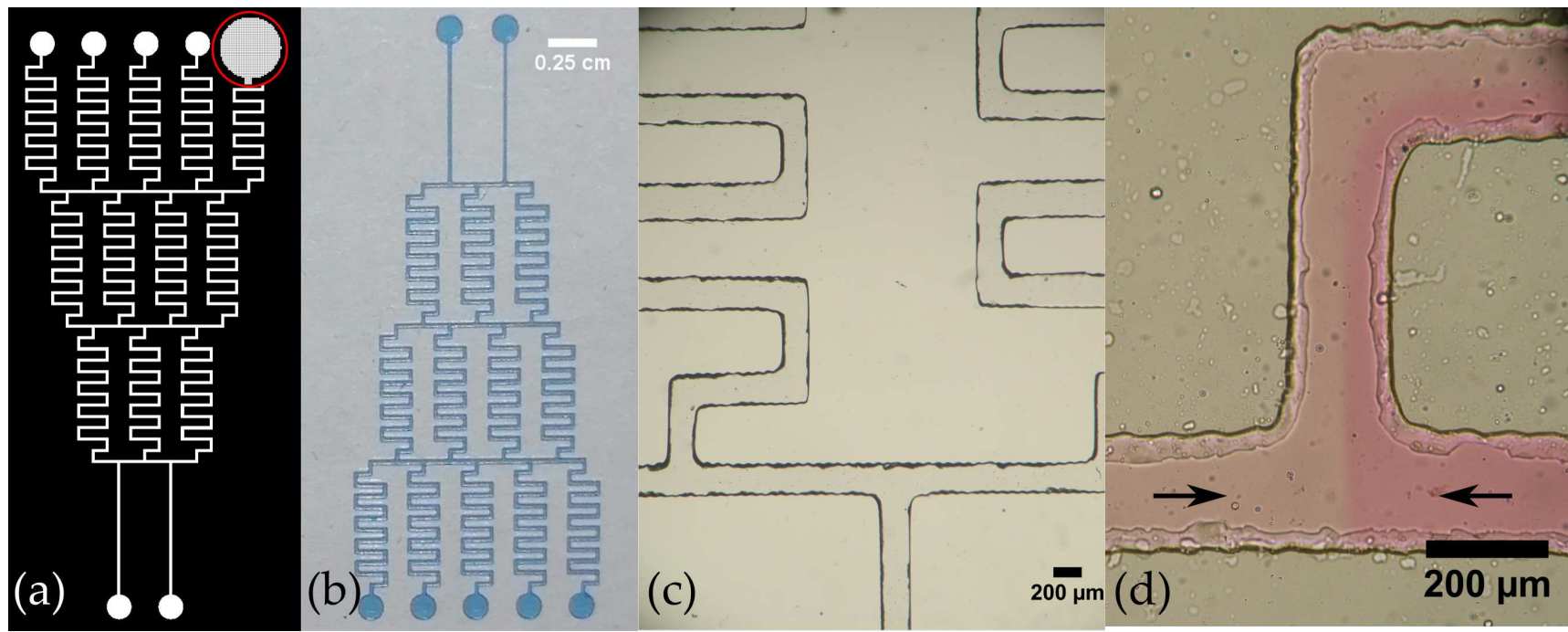

Fig. 5 a) Raw file used for the microchip design. The red circle is a magnified zone to show the drawing at the pixel level. b) Picture of the master mold made of dry film photoresist c) Optical image (x4 0.1NA) of the gradient mixer micro device filled with water. Channels are " 5 pixels" wide (i.e. $\approx 200 \mu \mathrm{m})$ and sidewall ripples are more prominent on the horizontal channel (d) Optical image (x10 $0.25 \mathrm{NA}$ ) of a junction of the gradient mixer where the concentrations $c_{0}$ and $c_{0} / 2$ start to mix by diffusion.

and increase the flexibility of fabrication of microfluidic devices.

Some techniques completely bypassed the use of photolithography, using direct methods of microfabrication : to cite a few examples, micromilling [17, lost wax [18, 3D printing [19, electrophoresis 20. Another perspective has focused on finding an easier and cheaper way to perform photolithography. Two major axis have been worked on. First of all, the use of resins (much easier to manipulate) have arisen with the development of so-called dryfilm photoresists. Originally introduced by Dupont in 1970, these films were initially used for printed circuit board (PCB) fabrication. These films have many advantages, including a short processing time and a uniform photoresist distribution 21. Moreover, the exposure energy is noticeably lower in this case, which means cheaper and more compact light sources such as commercial LEDs can be used 22. However, these techniques still require the engineering of high precision photomasks representing the circuits to be drawn on the resin. Two major drawbacks arise from this need : first of all, these high resolution masks are often produced by specific companies outside of the lab, and increase the overall cost and processing time of the whole operation. Secondly, new circuits will require new masks, which limits drastically the freedom of prototyping and the variety of the circuits that can be designed. Office printers can be used to print these masks 23 but at the cost of having a coarser $250 \mu \mathrm{m}$ resolution.

More recently, the overwhelming development of 3D printers and Computer-Aided Design (CAD) in gen- eral have been investigated to help with this idea of fast-prototyping microfluidic chips. The versatility of such instruments perfectly aligns with the problematic of 'fast prototyping'. Several techniques have been developed with this apparatus in mind. First of all, the microfluidic chip can be directly printed 2425 with specific resins, compatible with a wider range of chemical reagents [26, or transparent in order to be used within an optics framework 27] 28. However, these new materials are far less characterized compared to the very popular polymers such as PDMS (Polydimethylsiloxane) or PMMA (Polymethyl methacrylate). These polymers have now been used for a very long time, and they have become the flagship, and go-to materials for microfluidics 29 . As they have been studied and used for several decades now, these materials are far more flexible, and compatible with an incredibly wide range of chemicals. A middle-of-the-road solution would be to build a master mold using a 3D printer, instead of directly printing the circuit [19] 30. The microfluidic chip can then be obtained through soft lithography with well-characterized materials, such as those aforementioned.

The main line of the idea presented here is to have access to an out-of-the-box working setup to prepare microfluidic devices. For this matter, the use of a consumer grade SLA LCD 3D printer is relevant in many aspects. The device does not have to be modified in any way, avoiding thus any additional tedious step in the process. As an important side note, a very similar model (Phrozen Sonic MINI 4K) made by the same 
company, and using the same LCD screen and LED array is now available for $€ 350$ [10] and boasts many of the same characteristics, the only drawback being a smaller overall printing volume (which is not an issue for this photolithograpy application). It is safe to assume that the results presented here can be reproduced with this much cheaper SLA printer. Additionally, this reflects the current downward trend of consumer-grade SLA printer prices.

\section{Conclusion}

The system presented here is a compact, low cost (largely under $€ 1,000$ ), and turnkey system to manufacture microdevices master molds to be used with soft lithography afterwards. The convenient aspect of the method is that the $3 \mathrm{D}$ printer is usable out of the box for this purpose, without any further modification. Its undeniable flexibility is due to the direct design of patterns with a drawing software, pixel by pixel. Moreover, the entire master mold manufacturing process takes less than an hour.

The main concern compared to other stereolithography technologies like laser SLA or DLP, is the uneven pixel geometry of the LCD matrix that leads to sidewall ripples, mainly on horizontal channels. This aspect is only an issue for very small channels (1 and 2 pixels) and ultimately limits the resolution of the apparatus around $100 \mathrm{\mu m}$. However, commercial DLP or laser SLA setup for microfluidics lithography are at least one order of magnitude more expensive.

Creating chips is only one of the steps for performing microfluidic experiments, but one can also rely on other low cost alternatives for microfluidic pumps [31] 32, reser valves 34] or PDMS bonding 11. We believe that this system is a good entry point for labs wishing to start using microfluidics devices as tools for their experiment. It is also interesting for university/high school who wish to create introductory experimental labs on microfluidics.

Author contribution : Conceptualization, M.L.; project administration, M.L, D.G.; methodology, all authors; Data curation, V.C, T.T. , software, M.L., writing - original draft preparation, V.G. and M.L; writing - review and editing, all authors. All authors have read and agreed to the published version of the manuscript.

Funding This research was funded by ANR under Project ANR-17-CE09-0009 SIPAIE (Aggregation Induced Emission at Single-Particle level).

\section{Declarations}

Data availability The data sets supporting the results of this article are included within the article and its additional files (cf electronic supplementary material).

Ethical approval This article does not contain any studies with human participants or animals performed by any of the authors.

Consent to participate The authors consent to participate.

Consent to publish The authors consent to publish.

Competing interests The authors declare that they have no competing interests.

\section{References}

1. Neil Convery and Nikolaj Gadegaard. 30 Years of Microfluidics. Micro and Nano Engineering, 2(November 2018):76-91, 2019.

2. Yuksel Temiz, Robert D. Lovchik, Govind V. Kaigala, and Emmanuel Delamarche. Lab-on-a-chip devices: How to close and plug the lab? Microelectronic Engineering, 132:156-175, 2015.

3. Haimeng Pei, Lu Li, Zhaojun Han, Yiguo Wang, and Bo Tang. Recent advances in microfluidic technologies for circulating tumor cells: enrichment, single-cell analysis, and liquid biopsy for clinical applications. Lab Chip, pages -, 2020.

4. Yong Liu and Xingyu Jiang. Why microfluidics? Merits and trends in chemical synthesis. Lab on a Chip, 17(23):3960-3978, 2017.

5. Hoang Tuan Nguyen, Ha Thach, Emmanuel Roy, Khon Huynh, and Cecile Mong Tu Perrault. Low-cost, accessible fabrication methods for microfluidics research in lowresource settings. Micromachines, 9(9):1-10, 2018.

6. Jérôme Charmet, Rui Rodrigues, Ender Yildirim, Pavan Kumar Challa, Benjamin Roberts, Robert Dallmann, and Yudan Whulanza. Low-Cost microfabrication tool box. Micromachines, 11(2), 2020.

7. Jin Woo Lee, Shanna R. Daly, Aileen Y. Huang-Saad,

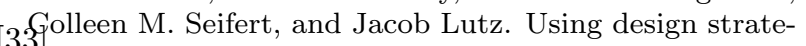
gies from microfluidic device patents to support idea generation. Microfluidics and Nanofluidics, 22(7):1-22, 2018.

8. Bruce K. Gale, Alexander R. Jafek, Christopher J. Lambert, Brady L. Goenner, Hossein Moghimifam, Ugochukwu C. Nze, and Suraj Kumar Kamarapu. A review of current methods in microfluidic device fabrication and future commercialization prospects. Inventions, 3(3), 2018.

9. Fatemeh Khalkhal, Kendrick H. Chaney, and Susan J. Muller. Optimization and application of dry film photoresist for rapid fabrication of high-aspect-ratio microfluidic devices. Microfluidics and Nanofluidics, 20(11):153, 2016.

10. Phrozen 3d lcd printers. https://www.phrozen3dp.com/ categories/phrozen-lcd-3d-printer. Accessed: 202102-16.

11. Kathryn Haubert, Tracy Drier, and David Beebe. Pdms bonding by means of a portable, low-cost corona system. Lab on a Chip, 6(12):1548-1549, 2006. 
12. Martinus H.V. Werts, Vincent Raimbault, Rozenn Texier-Picard, Rémi Poizat, Olivier Franais, Laurent Griscom, and Julien R.G. Navarro. Quantitative fullcolour transmitted light microscopy and dyes for concentration mapping and measurement of diffusion coefficients in microfluidic architectures. Lab on a Chip, 12(4):808-820, 2012.

13. Xiang Wang, Zhaomiao Liu, and Yan Pang. Concentration gradient generation methods based on microfluidic systems. RSC Advances, 7(48):29966-29984, 2017.

14. Yi Wang, Tamal Mukherjee, and Qiao Lin. Systematic modeling of microfluidic concentration gradient generators. Journal of Micromechanics and Microengineering, 16(10):2128-2137, 2006.

15. Kwang W. Oh, Kangsun Lee, Byungwook Ahn, and Edward P. Furlani. Design of pressure-driven microfluidic networks using electric circuit analogy. Lab on a Chip, 12(3):515-545, 2012.

16. Stephan K. W. Dertinger, Daniel T. Chiu, Noo Li Jeon, and George M. Whitesides. Generation of Gradients Having Complex Shapes Using Microfluidic Networks. Analytical Chemistry, 73(6):1240-1246, mar 2001.

17. David J. Guckenberger, Theodorus E. De Groot, Alwin M.D. Wan, David J. Beebe, and Edmond W.K. Young. Micromilling: A method for ultra-rapid prototyping of plastic microfluidic devices. Lab on a Chip, 15(11):2364-2378, 2015.

18. Chunhui Chung, Yann Jiun Chen, Pin Chaun Chen, and Chia Yuan Chen. Fabrication of PDMS passive micromixer by lost-wax casting. International Journal of Precision Engineering and Manufacturing, 16(9):2033$2039,2015$.

19. Sajad Razavi Bazaz, Navid Kashaninejad, Shohreh Azadi, Kamal Patel, Mohsen Asadnia, Dayong Jin, and Majid Ebrahimi Warkiani. Rapid Softlithography Using 3D-Printed Molds. Advanced Materials Technologies, 4(10):1-11, 2019.

20. Raphael Renaudot, Yves Fouillet, Laurent Jalabert, Momoko Kumemura, Dominique Collard, Hiroyuki Fujita, and Vincent Agache. Programmable LDEP technology to fabricate versatile master molds for PDMS continuous-flow microfluidic applications. Microfluidics and Nanofluidics, 16(4):701-710, 2014.

21. P. Vulto, N. Glade, L. Altomare, J. Bablet, L. Del Tin, G. Medoro, I. Chartier, N. Manaresi, M. Tartagni, and R. Guerrieri. Microfluidic channel fabrication in dry film resist for production and prototyping of hybrid chips. Lab on a Chip, 5(2):158, 2005.

22. B. G.C. Maisonneuve, T. Honegger, J. Cordeiro, O. Lecarme, T. Thiry, D. Fuard, K. Berton, E. Picard, M. Zelsmann, and D. Peyrade. Rapid mask prototyping for microfluidics. Biomicrofluidics, 10(2), 2016.

23. Tao Deng, Hongkai Wu, Scott T. Brittain, and George M. Whitesides. Prototyping of masks, masters, and stamps/molds for soft lithography using an office printer and photographic reduction. Analytical Chemistry, 72(14):3176-3180, 2000.

24. Sidra Waheed, Joan M. Cabot, Niall P. Macdonald, Trevor Lewis, Rosanne M. Guijt, Brett Paull, and Michael C. Breadmore. 3D printed microfluidic devices: Enablers and barriers. Lab on a Chip, 16(11):1993-2013, 2016.

25. Zongjie Wang, Nicholas Martin, Delator Hini, Barry Mills, and Keekyoung Kim. Rapid fabrication of multilayer microfluidic devices using the liquid crystal displaybased stereolithography 3D printing system. 3D Printing and Additive Manufacturing, 4(3):156-164, 2017.
26. Frederik Kotz, Patrick Risch, Dorothea Helmer, and Bastian E. Rapp. High-Performance Materials for 3D Printing in Chemical Synthesis Applications. Advanced Materials, 31(26), 2019.

27. Anthony K. Au, Wonjae Lee, and Albert Folch. Mailorder microfluidics: Evaluation of stereolithography for the production of microfluidic devices. Lab on a Chip, 14(7):1294-1301, 2014.

28. Frederik Kotz, Karl Arnold, Stefan Wagner, Werner Bauer, Nico Keller, Tobias M. Nargang, Dorothea Helmer, and Bastian E. Rapp. Liquid PMMA: A High Resolution Polymethylmethacrylate Negative Photoresist as Enabling Material for Direct Printing of Microfluidic Chips. Advanced Engineering Materials, 20(2):1-5, 2018.

29. Kiran Raj M and Suman Chakraborty. PDMS microfluidics: A mini review. Journal of Applied Polymer Science, 137(27):1-14, 2020.

30. Mohamed G.A. Mohamed, Hitendra Kumar, Zongjie Wang, Nicholas Martin, Barry Mills, and Keekyoung Kim. Rapid and inexpensive fabrication of multi-depth microfluidic device using high-resolution LCD stereolithographic 3D printing. Journal of Manufacturing and Materials Processing, 3(1):1-11, 2019.

31. Philipp Frank, Sebastian Haefner, Martin Elstner, and Andreas Richter. 2016 Fully-Programmable, Low-Cost, " Do-It-Yourself " Pressure Source for General Purpose Use in the Microfluidic Laboratory. Inventions, 1(2):111, 2016.

32. Peter Thurgood, Sergio Aguilera Suarez, Sheng Chen, Christopher Gilliam, Elena Pirogova, Aaron R. Jex, Sara Baratchi, and Khashayar Khoshmanesh. Self-sufficient, low-cost microfluidic pumps utilising reinforced balloons. Lab on a Chip, 19(17):2885-2896, 2019.

33. Direct delivery of reagents from a pipette tip to a pdms microfluidic device. https: //blogs.rsc.org/chipsandtips/2015/10/09/ direct-delivery-of-reagents-from-a-pipette-tip-to-a-pdms-micr Accessed: 2020-10-14. 34. A simple microfluidic 4-way valve by
clamping interconnected tubing. //blogs.rsc.org/chipsandtips/2011/10/17/ a-simple-microfluidic-4-way-valve-by-clamping-interconnectedAccessed: 2020-10-14. 


\section{Figures}

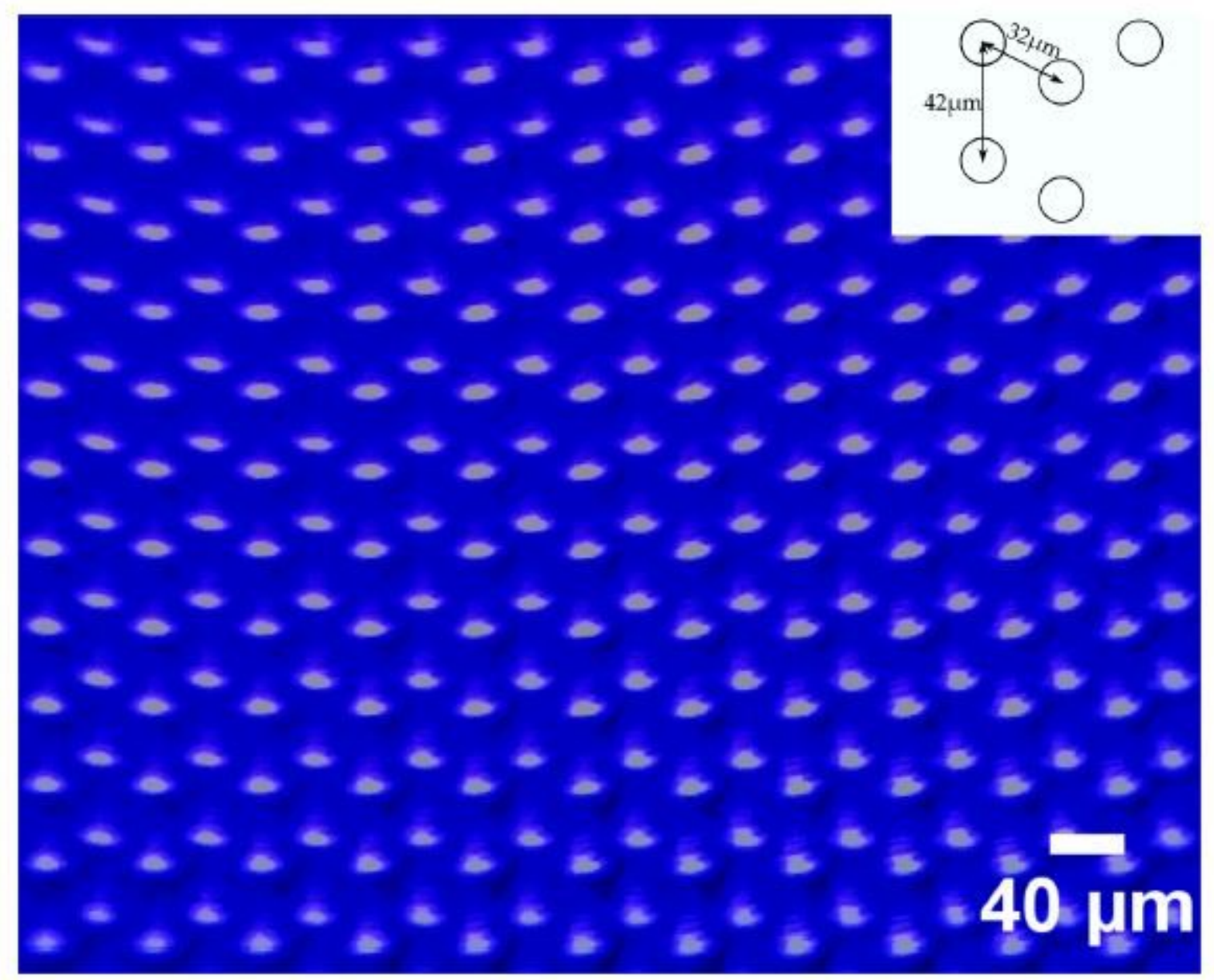

Figure 1

Image of the LCD matrix used for illuminating the photoresist Im. Inset : schematic of the pixel geometry of the LCD matrix. 


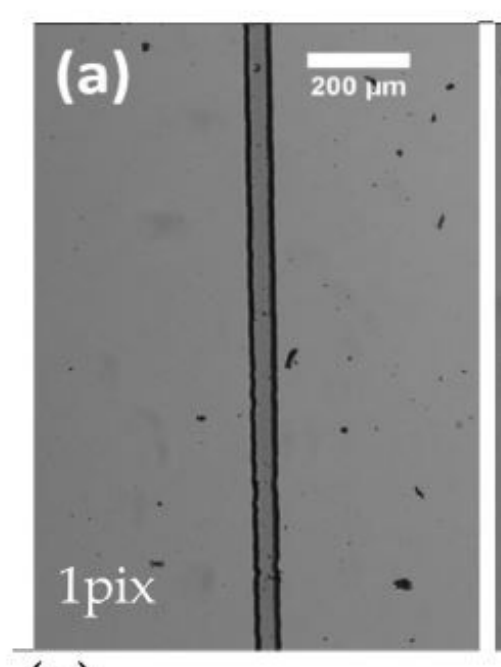

(e)

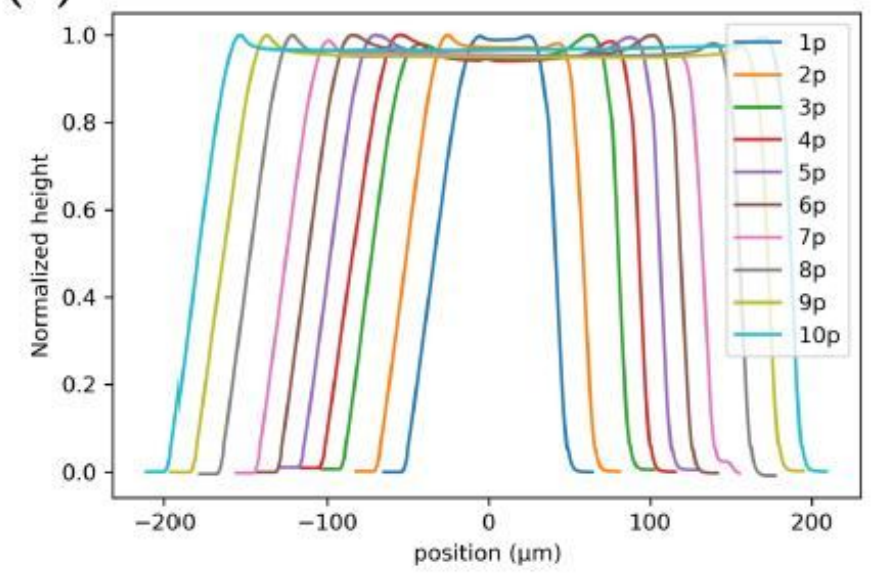

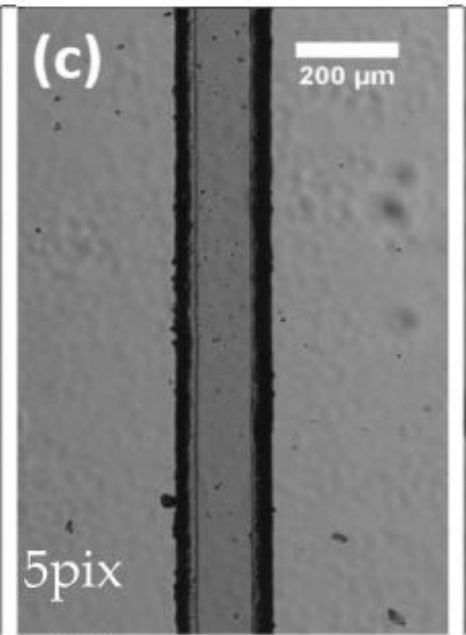

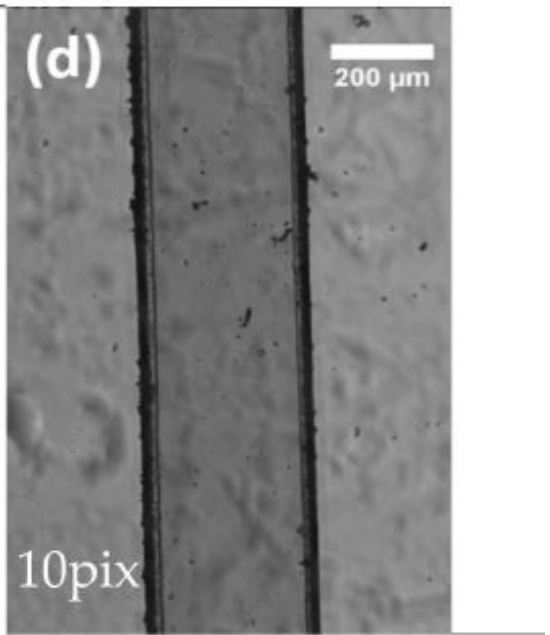

(f)

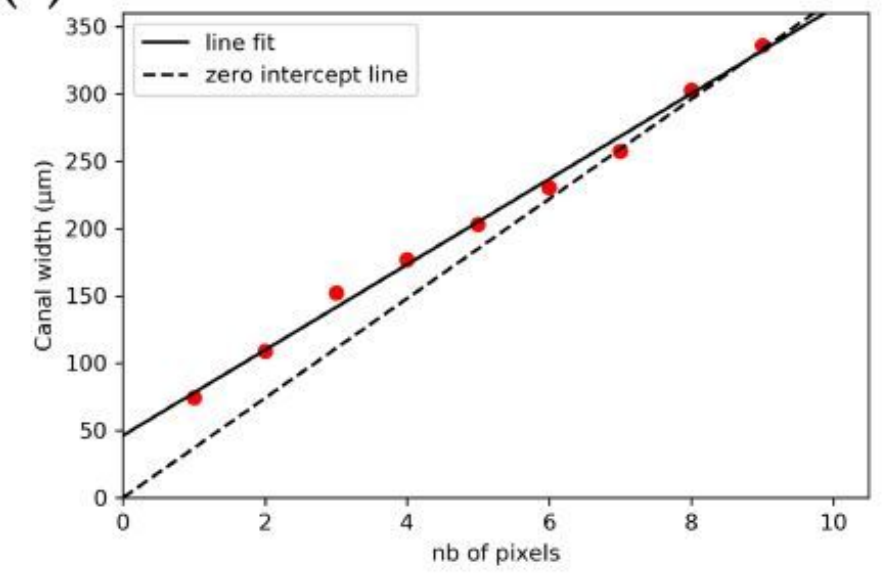

Figure 2

a,b,c,d) Optical image of a micro-channel (vertically insolated) obtained with 1, 2, 5 and 10 pixels of the LCD screen respectively. e) Horizontal prole of micro-channels obtained for a width corresponding from 1 to 10 pixels of the LCD screen. f) Evolution of the width at half maximum of vertical micro-channels with the number of pixels on the LCD screen. The plain line takes into account all the points whereas the dashed line has a forced zero intercept and only takes into account the channels made with more than 5 pixels. 


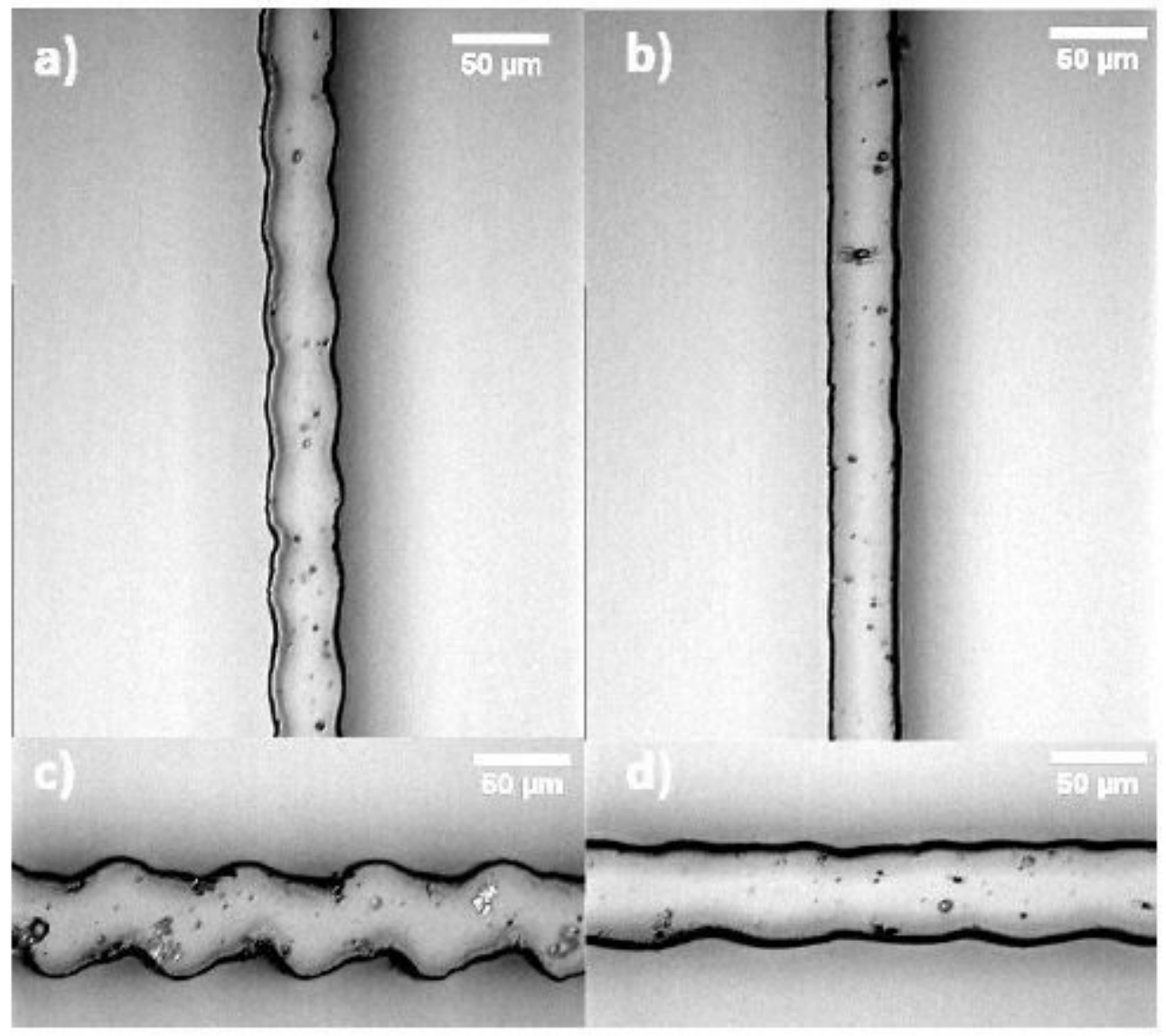

Figure 3

Optical image of a micro-channel created from 1 pixel of a LCD screen a) vertical, pressed against the LCD screen. The small sidewall ripples may be attributed to the fact that the vertical pixels of the LCD matrix are not totally contiguous. b) vertical, insolated $340 \mu \mathrm{m}$ away from the LCD screen, c) horizontal, pressed against the LCD screen. The important sidewall ripples are most surely due to the fact that, for drawing a horizontal line, alternating pixels of two different rows are turned on by the LCD matrix. (cf $\mathrm{g} 1$ ). d) horizontal, insolated $340 \mu \mathrm{m}$ away from the LCD screen 


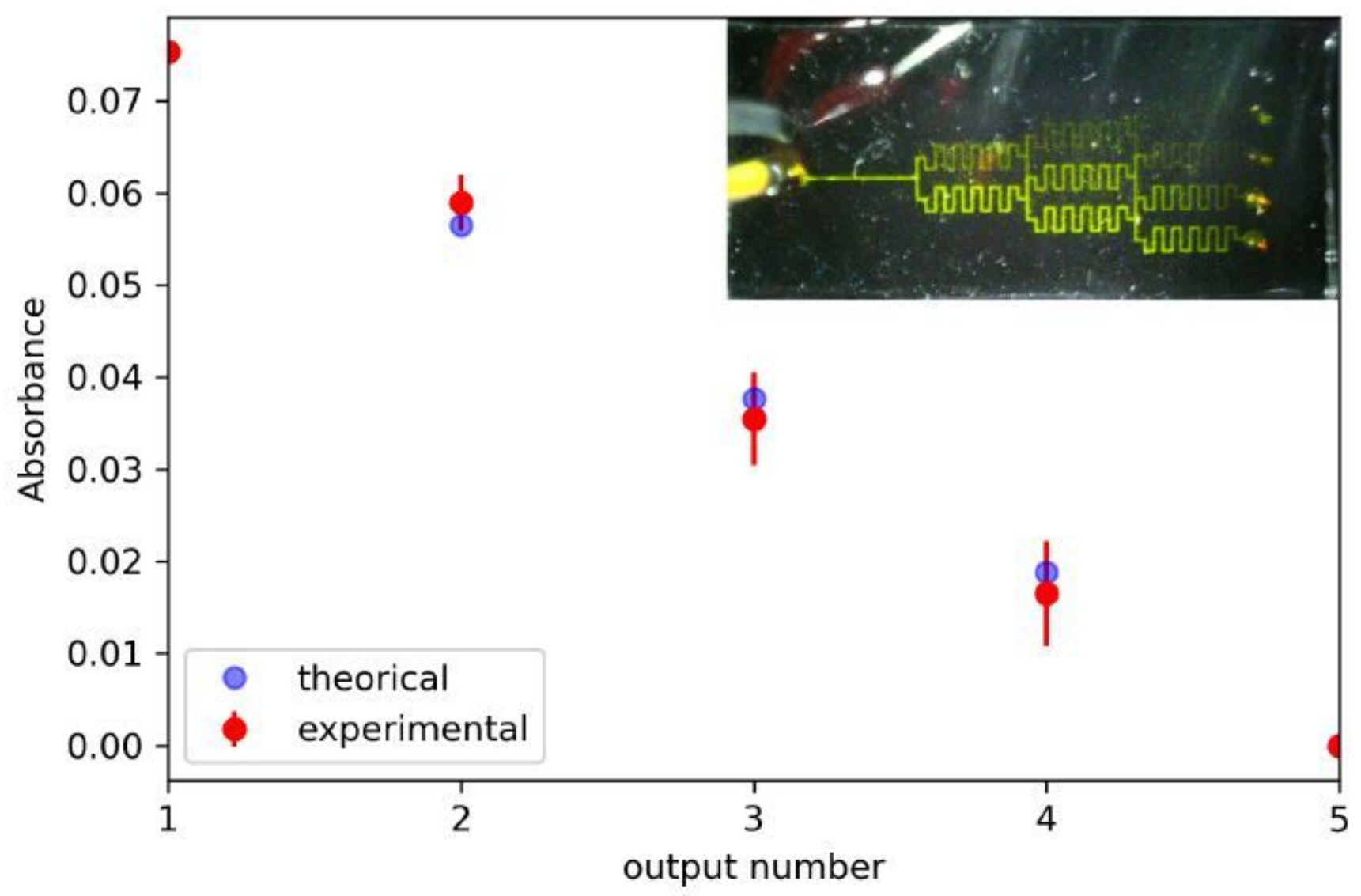

Figure 4

Absorbance obtained at the five outputs of the gradient mixer chip after mixing Rhodamine $6 \mathrm{G}$ and water. Inset: picture of the microdevice with Rhodamine $6 \mathrm{G}$ visualised via its uorescence.
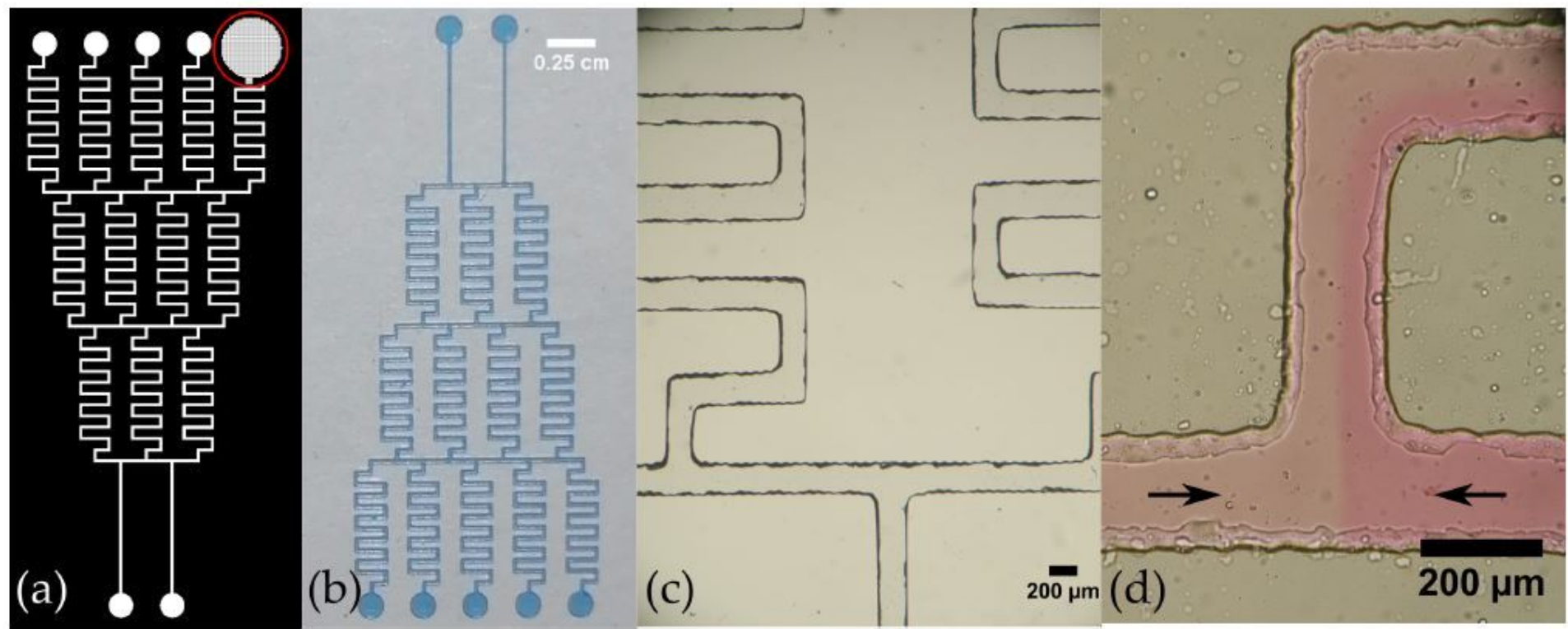

Figure 5 
a) Raw le used for the microchip design. The red circle is a magnied zone to show the drawing at the pixel level. b) Picture of the master mold made of dry Im photoresist c) Optical image ( $x 40.1 \mathrm{NA}$ ) of the gradient mixer micro device lled with water. Channels are " 5 pixels" wide (i.e. $\approx 200 \mu \mathrm{m}$ ) and sidewall ripples are more prominent on the horizontal channel (d) Optical image ( $x 100.25 \mathrm{NA})$ of a junction of the gradient mixer where the concentrations $\mathrm{c} 0$ and $\mathrm{c} 0=2$ start to mix by diffusion.

\section{Supplementary Files}

This is a list of supplementary files associated with this preprint. Click to download.

- SIColinetal.pdf 